\title{
A note on Zagreb indices inequality for trees and unicyclic graphs*
}

\author{
Vesna Andova \\ Faculty of Electrical Engineering and Information Technologies \\ Ss Cyril and Methodius Univ., Ruger Boskovik PO Box 574, 1000 Skopje, Macedonia \\ Nathann Cohen ${ }^{\dagger}$ \\ Projet Mascotte, I3S (CNRS, UNSA) and INRIA, 2004 route des Lucioles \\ BP 93, 06902 Sophia-Antipolis Cedex, France \\ Riste Škrekovski ‡ \\ Department of Mathematics, University of Ljubljana \\ Jadranska 21, 1111 Ljubljana, Slovenia
}

Received 10 September 2010, accepted 8 December 2010, published online 2 November 2011

\begin{abstract}
For a simple graph $G$ with $n$ vertices and $m$ edges, the inequality $\frac{M_{1}(G)}{n} \leq \frac{M_{2}(G)}{m}$, where $M_{1}(G)$ and $M_{2}(G)$ are the first and the second Zagreb indices of $G$, is known as Zagreb indices inequality. Recently Vukičević and Graovac [12], and Caporossi, Hansen and Vukičević [3] proved that this inequality holds for trees and unicyclic graphs, respectively. Here, alternative and shorter proofs of these results are presented.
\end{abstract}

Keywords: First Zagreb index, second Zagreb index.

Math. Subj. Class.: 05C05, 05C07, 05C38, 92E10, 94C15

\section{Introduction}

The first and second Zagreb indices are among the oldest topological indices, defined in 1972 by Gutman et al. [5], and are given different names in the literature, such as the Zagreb group indices, the Zagreb group parameters and most often, the Zagreb indices.

\footnotetext{
* Partial support by bilateral project BI-FR/09-10-PROTEUS-008.

${ }^{\dagger}$ Partially supported by the ANR Blanc AGAPE and ANR Blanc International-Taiwan GRATEL.

${ }^{\ddagger}$ Supported in part by ARRS Research Program P1-0297.

E-mail addresses: vesna.andova@gmail.com (Vesna Andova), nathann.cohen@gmail.com (Nathann Cohen), skrekovski@gmail.com (Riste Škrekovski)
} 
Since then, they have been used to study molecular complexity, chirality, ZE-isomerism and hetero-systems (see [1, 4, 8, 10, 14]).

In the following, let $G=(V, E)$ be a simple graph with $n=|V|$ vertices and $m=|E|$ edges. These indices are defined as

$$
M_{1}(G)=\sum_{v \in V} d(v)^{2} \quad \text { and } \quad M_{2}(G)=\sum_{u v \in E} d(u) d(v),
$$

where $d(u)$ stands for the degree of vertex $u$. For the sake of simplicity, we will often use $M_{1}$ and $M_{2}$ instead of $M_{1}(G)$ and $M_{2}(G)$, respectively.

In 2003, an article [9] repopularized Zagreb indices, and since then a lot of work was done on this topic. For more results concerning Zagreb indices see [7, 13]. Comparing the values of these indices on the same graph was one very natural aim, which gave, and still gives, very interesting results. At first the next conjecture was proposed [2]:

Conjecture 1.1. For all simple graphs $G$,

$$
\frac{M_{1}(G)}{n} \leq \frac{M_{2}(G)}{m}
$$

and the bound is tight for complete graphs.

If the graph is regular then this bound is tight, but it is also tight if $G$ is a star. This inequality holds for trees [12], graphs of maximum degree four, i.e. so called chemical graphs [6] and unicyclic graphs [3], but does not hold in general. See [6, 12, 3, 11] for various examples of graphs dissatisfying the inequality (1.1).

For a connected graph $G$, the cyclomatic number is $\nu(G)=m-n+1$. Thus, every tree has cyclomatic number 0 . A graph whose cyclomatic number is 1 is called unicyclic. Note that such a graph has precisely one cycle.

In chemistry trees, unicyclic graphs, bicyclic graphs, and so on, are very important graphs since they represent classes of molecules. Trees are graph representation of acyclic molecules like alkanes (also known as paraffins). Cycloalkanes are types of alkanes which have one or more rings of carbon atoms in the chemical structure of their molecules, so their graphs are unicyclic graphs, bicyclic graphs, etc.

In this paper we present alternative proofs concerning the Zagreb indices inequality for trees and unicyclic graphs.

\section{An alternative proof for trees and unicyclic graphs}

As we said before, trees and unicyclic graphs satisfy $M_{1} / n \leq M_{2} / m$. Here, these results are proven in a shorter way.

A star with $k$ edges is called a $k$-star. A path of length $k$ is called a $k$-path. Let $p_{3}(G)$ be the number of 3-paths, $p_{2}(G)$ the number of 2-paths, and $C_{3}(G)$ is the number of 3-cycles in $G$. Note that

$$
p_{3}(G)+3 C_{3}(G)=\sum_{u v \in E}(d(v)-1)(d(u)-1),
$$

where $u v$ in the summation is the middle edge of the $(d(u)-1)(d(v)-1)$ corresponding 3 -paths. Obviously, a 3-path corresponds to a 3-cycle when its endvertices coincide. 
Theorem 2.1. For any tree $G \neq K_{1}$, it holds $\frac{M_{1}}{n} \leq \frac{M_{2}}{m}$. Moreover, equality holds if and only if $G$ is a star.

Proof. If $G$ is a $k$-star, then $M_{1}=k n$ and $M_{2}=k m$, by which we have equality in (1.1). So assume now that $G$ has at least two internal adjacent vertices $u$ and $v$ and that $v$ is the only internal neighbor of $u$. Observe that $M_{1}=\sum_{v \in V} d(v)^{2}=2\left(p_{2}(G)+m\right)$. We have

$$
M_{2}=\sum_{u v \in E}[(d(v)-1)(d(u)-1)+(d(u)+d(v))-1]=p_{3}(G)+M_{1}-m
$$

Now, since $m=n-1$, we obtain

$$
\begin{aligned}
(n-1) M_{1} & <n M_{2} \\
(n-1) M_{1} & <n\left[p_{3}(G)+M_{1}-(n-1)\right] \\
0 & <p_{3}(G)+\frac{2}{n}\left(p_{2}(G)+(n-1)\right)-(n-1) .
\end{aligned}
$$

Notice that $p_{2}(G) \geq 2$ for every tree on at least 4 vertices. Now, we will prove that $p_{3}(G) \geq n-3$, and this will establish the theorem. Let $l_{1}, \ldots, l_{k}$ be the leaves adjacent to $u$, and let $w \neq u$ be a neighbor of $v$. To any vertex $x$ at distance at least 2 from $u$ we associate the 3-path built from the first three edges of the shortest path from $x$ to $l_{1}$. To any leaf $l_{i},(i \neq 1)$, we associate the path from $w$ to $l_{i}$. These 3 -paths being all different, we associated a 3 -path to any vertex except three, namely $l_{1}, u, v$, which ensures that $p_{3}(G) \geq n-3$.

Theorem 2.2. For any unicyclic graph $G$, it holds $\frac{M_{1}}{n} \leq \frac{M_{2}}{m}$. Moreover, equality holds if and only if $G$ is a cycle.

Proof. Since $G$ is an unicyclic graph, $m=n$, and so we need to show $M_{1} \leq M_{2}$. If $G$ is a $k$-cycle then $M_{1}=4 k=M_{2}$, and we have equality in (1.1). So, assume that $G$ is not a cycle, $C=x_{1} x_{2} \cdots x_{l} x_{1}$ is the unique cycle of $G$ and $x_{1}$ has a neighbor $y \notin V(C)$. From (2.1) and the left equality of (2.2), we have

$$
M_{2}=p_{3}(G)+3 C_{3}(G)+M_{1}-m .
$$

It is enough to show that $M_{1}+1 \leq M_{2}$ which is equivalent to $M_{1} \leq p_{3}(G)+3 C_{3}(G)+$ $M_{1}-n-1$, and hence is equivalent to

$$
n+1 \leq p_{3}(G)+3 C_{3}(G) .
$$

Now, remove the edge $x_{1} x_{2}$ from the cycle. Then $G-x_{1} x_{2}$ is a tree and $p_{3}\left(G-x_{1} x_{2}\right) \geq$ $n-3$. Including $y x_{1} x_{2} x_{3}$ we have at least $n-2$ different 3 -paths.

If $C$ is a 3 -cycle, then it is obvious that (2.3) holds. Now, assume $l \geq 4$. Observe that $x_{1} x_{2} x_{3} x_{4}, x_{l} x_{1} x_{2} x_{3}, x_{l-1} x_{l} x_{1} x_{2}$ are 3-paths all distinct from the 3-paths described. Hence, $p_{3}(G) \geq n+1$. This implies (2.3). 


\section{References}

[1] D. Bonchev, N. Trinajstić, Overall Molecular Descriptors, 3, Overall Zagreb Indices, SAR\&QSAR Environ. Res. 12 (2001), 213-236.

[2] G. Caporossi and P. Hansen, Variable Neighborhood search for extremal graphs, 1, The AutoGraphiX system, Discrete Math. 212 (2000), 29-44.

[3] G. Caporossi, P. Hansen and D. Vukičević, Comparing Zagreb indices of cyclic graphs, MATCH Commun. Math. Comput. Chem. 63 (2010), 441-451.

[4] I. Gutman, B. Rušćić, N. Trinajstić and C. F. Wilcox, Jr., Graph Theory and Molecular Orbitals, XII, Acyclic Polyenes, J. Chem. Phys. 62 (1975), 3399-3405.

[5] I. Gutman and N. Trinajstić, Graph theory and molecular orbitals, Total $\pi$-electron energy of alternant hydrocarbons, Chem. Phys. Lett. 17 (1972), 535-538.

[6] P. Hansen and D. Vukičević, Comparing the Zagreb Indices, Croat. Chem. Acta 80 (2007), $165-168$.

[7] B. Liu and Z. You, A survey on comparing Zagreb indices, in: I. Gutman, B. Furtula (eds), Novel Molecular Structure Descriptors - Theory and Applications I, Univ. Kragujevac, Kragujevac, 2010, 227-239.

[8] A. Miličević, S. Nikolić and N. Trinajstić, On Reformulated Zagreb Indices, Molecular Diversity 8 (2004), 393-399.

[9] S. Nikolić, G. Kovačević, A. Miličević and N. Trinajstić, The Zagreb Indices 30 years after, Croat. Chem. Acta 76 (2003), 113-124.

[10] S. Nikolić, I. M. Tolić, N. Trinajstić and I. Baučic, On the Zagreb Indices as Complexity Indices, Croat. Chem. Acta 73 (2000), 909-921.

[11] L. Sun and S. Wei, Comparing the Zagreb indices for connected bicyclic graphs, MATCH Commun. Math. Comput. Chem. 62 (2009), 699-714.

[12] D. Vukičević and A. Graovac, Comparing Zagreb $M_{1}$ and $M_{2}$ indices for acyclic molecules, MATCH Commun. Math. Comput. Chem. 57 (2007), 587-590.

[13] D. Vukičević and A. Graovac, Comparing Zagreb $M_{1}$ and $M_{2}$ indices: Overview of the results, manuscript.

[14] D. Vukičević, S. Nikolić and N. Trinajstić, On the Path-Zagreb Matrix, J. Math. Chem. 45 (2009), 538-543. 